Supporting information (SI)

\title{
Electronic Decoupling of Organic Layers by a Self-assembled Supramolecular Network on $\mathrm{Au}(111)$
}

Zhonghua Liu ${ }^{1}$, Kewei Sun ${ }^{1}$, Xuechao Li, Ling Li, Haiming Zhang* and Lifeng Chi*

AUTHOR ADDRESS

Institute of Functional Nano \& Soft Materials (FUNSOM), Jiangsu Key

Laboratory for Carbon-Based Functional Materials \& Devices, Soochow

University, 199 Ren'ai Road, Suzhou, 215123, Jiangsu, PR China

AUTHOR INFORMATION

Corresponding Author

*Haiming Zhang: hmzhang@suda.edu.cn

*Lifeng Chi: chilf@suda.edu.cn 
The thermal stability of the CA·M layer.

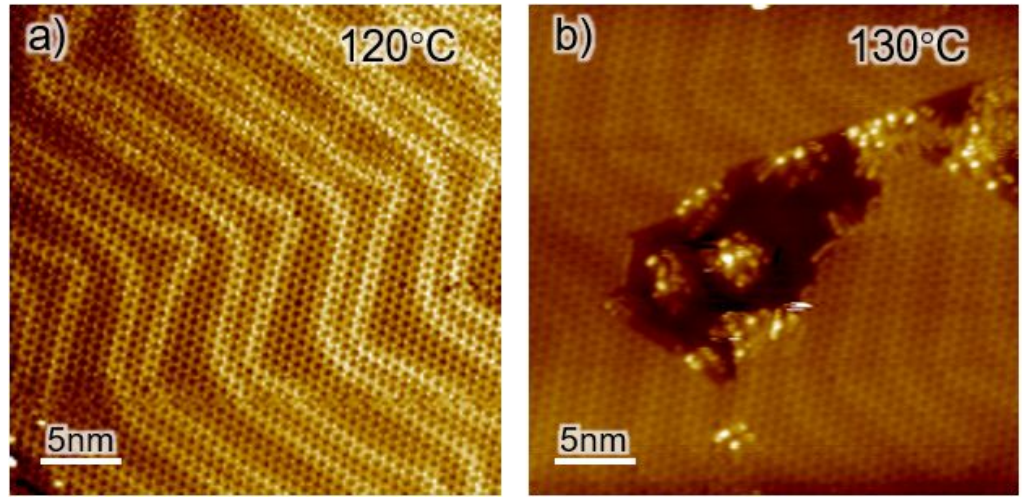

Figure S1. The structure of CA.M layer at different temperatures. a) $120^{\circ} \mathrm{C}$. b) $130^{\circ} \mathrm{C}$. 